\title{
Heating of trapped ions from the quantum ground state
}

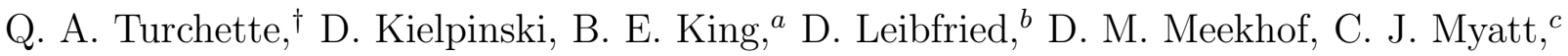

M. A. Rowe, C. A. Sackett, C. S. Wood, W. M. Itano, C. Monroe, and D. J. Wineland

Time and Frequency Division, National Institute of Standards and Technology, Boulder, CO

80303

(October 24, 2018)

We have investigated motional heating of laser-cooled ${ }^{9} \mathrm{Be}^{+}$ions held in radio-frequency (Paul) traps. We have measured heating rates in a variety of traps with different geometries, electrode materials, and characteristic sizes. The results show that heating is due to electric-field noise from the trap electrodes which exerts a stochastic fluctuating force on the ion. The scaling of the heating rate with trap size is much stronger than that expected from a spatially uniform noise source on the electrodes (such as Johnson noise from external circuits), indicating that a microscopic uncorrelated noise source on the electrodes (such as fluctuating patch-potential fields) is a more likely candidate for the source of heating.

\section{INTRODUCTION}

Cold trapped ions have been proposed as a physical implementation for quantum computation (QC) [1], and experiments on one [2 [5] and two [6.7] ions have demonstrated proof of the principle. Work is currently underway to extend these results. In ion trap QC, ion-laser interactions prepare, manipulate and entangle atomic states in ways dependent on the quantum motional state of the ions. A limiting factor in the fidelity of an operation is uncontrolled heating of the motion during manipulations. Heating leads to decoherence of the quantum superposition states involved in the computation [8.9], and can ultimately limit the number of elementary gate operations which can be strung together. Speculations 
have been made about the mechanisms that lead to heating [8,10 [14], but measurements are scarce since the necessary sensitivity can be achieved only through laser cooling to near the ground state of motion. Additionally, systematic studies of the dependence of heating rate on various trap properties are difficult, since often this requires the construction and operation of an entirely new trap apparatus which may have different values of properties not under study. Indeed, the data presented here pose several interpretational difficulties for this reason.

Heating of a single trapped ion (or the center-of-mass motion of a collection of trapped ions) occurs when noisy electric fields at the position of the ion couple to its charge, giving rise to fluctuating forces. If the spectrum of fluctuations overlaps the trap secular motion frequency or its micro-motion sidebands, the fluctuating forces can impart significant energy to the secular motion of the ion. Here, we express the heating rate as the average number of quanta of energy gained by the secular motion in a given time. There are several candidates worth considering for sources of the noisy fields which give rise to heating. Some of these are 88: Johnson noise from the resistance in the trap electrodes or external circuitry (the manifestation of thermal electronic noise or black body radiation consistent with the boundary conditions imposed by the trap electrode structure), fluctuating patch-potentials (due, for example, to randomly oriented domains at the surface of the electrodes or adsorbed materials on the electrodes), ambient electric fields from injected electronic noise, fields generated by fluctuating currents such as electron currents from field-emitter points on the trap electrodes, and collisions with background atoms. Only the first two mechanisms will be considered here since the remaining mechanisms (and others) are unlikely contributers [8] or can be eliminated by comparing the measured heating rates of the center-of-mass and differential modes of two ions [6]. As will be shown below, the Johnson-noise and patch-potential mechanisms give rise to heating rates which scale differently with the distance between the ion and the trap electrodes. 


\section{TWO MODELS FOR SOURCES OF HEATING}

\section{A. Preliminaries}

The heating rate caused by a fluctuating uniform field can be derived as in Savard, et al. [15] and agrees with a classical calculation [8]. The Hamiltonian for a particle of charge $q$ and mass $m$ trapped in an harmonic well subject to a fluctuating, uniform (non-gradient) electric field drive $\epsilon(t)$ is

$$
H(t)=H_{0}-q \epsilon(t) x
$$

where $H_{0}=p^{2} / 2 m+m \omega_{m}^{2} x^{2}$ is the usual, stationary harmonic oscillator Hamiltonian with trap frequency $\omega_{m}$. From first order perturbation theory, the rate of transition from the ground state of the well $(|n=0\rangle)$ to the first excited state $(|n=1\rangle)$ is 15

$$
\Gamma_{0 \rightarrow 1}=\frac{1}{\hbar^{2}} \int_{-\infty}^{\infty} d \tau e^{i \omega_{m} \tau}\langle\epsilon(t) \epsilon(t+\tau)\rangle|\langle 0|q x| 1\rangle|^{2}
$$

Evaluating the motional matrix element gives

$$
\Gamma_{0 \rightarrow 1}=\frac{q^{2}}{m \hbar \omega_{m}} S_{E}\left(\omega_{m}\right)
$$

where $S_{E}(\omega) \equiv \int_{-\infty}^{\infty} d \tau e^{i \omega_{m} \tau}\langle\epsilon(t) \epsilon(t+\tau)\rangle$ is the spectral density of electric-field fluctuations.

For an ion trapped by a combination of (assumed noiseless) static and inhomogeneous rf fields (Paul trap) the heating rate can be generalized to [11:

$$
\dot{\bar{n}}=\frac{q^{2}}{4 m \hbar \omega_{m}}\left(S_{E}\left(\omega_{m}\right)+\frac{\omega_{m}^{2}}{2 \Omega_{T}^{2}} S_{E}\left(\Omega_{T} \pm \omega_{m}\right)\right),
$$

where $\dot{\bar{n}}$ is the rate of change of the average thermal occupation number, $\omega_{m}$ is now the secular frequency of the mode of motion under consideration and $\Omega_{T}$ is the trap rf-drive frequency. The second term on the rhs of Eq. 1 is due to a cross-coupling between the rf and noise fields; it will not be present for the axial motion of a linear trap which is confined only by static fields. Even for motion confined by rf pondermotive forces, this second term 
will be negligible in the absence of spurious resonances in $S_{E}(\omega)$ or increasing $S_{E}(\omega)$ (since $\omega_{m}^{2} / \Omega_{T}^{2} \sim 10^{-4}$ ) and is neglected in what follows 8 .

We differentiate two sources of the noise that gives rise to heating. The first is thermal electronic noise in the imperfectly conducting trap electrodes and elsewhere in the trap circuitry. Though this source of noise is ultimately microscopic in origin, for our purposes here it can be treated adequately by use of lumped circuit models. Thermal noise has been considered in the context of ion-trap heating in several places [8,11, 14. The second source of noise considered here is due to "microscopic" regions of material (small compared to the size of the trap electrodes) with fluctuating, discontinuous potentials established, for example, at the interface of different materials or crystalline domains. We call this patch-potential noise, and its microscopic origin leads to manifestly different heating behaviour from that for the thermal electronic noise case. Static patch-potentials are a well-known phenomenon, but little is known about the high-frequency $(\mathrm{MHz})$ fluctuating patches which are required to account for our observed heating rates [16 [18].

\section{B. Thermal electronic noise}

Heating rates in the case of thermal electronic noise (Johnson noise) can be obtained simply through the use of lumped-circuit models, which are justified by the fact that the wavelength of the relevant fields (at typical trap secular or drive frequencies) is significantly larger than the size of the trap electrodes. Such an analysis has been carried out elsewhere [8,11, 19], and only the major results will be quoted here. Resistances in the trap electrodes and connecting circuits give rise to an electric field noise spectral density $S_{E}(\omega)=4 k_{B} T R(\omega) / d^{2}$ where $d$ is the characteristic distance from the trap electrodes to the ion, $T$ is the temperature (near room temperature for all of our experiments), $k_{B}$ is

Boltzmann's constant, and $R(\omega)$ is the effective (lumped-circuit) resistance between trap electrodes. The heating rate is given by

$$
\dot{\bar{n}}_{\mathrm{R}}=\frac{q^{2} k_{B} T R\left(\omega_{m}\right)}{m \hbar \omega_{m} d^{2}} .
$$


A numerical estimate of the heating rate for typical trap parameters gives $0.1 / \mathrm{s}<\dot{\bar{n}}_{\mathrm{R}}<1 / \mathrm{s}$ [8.11], which is significantly slower than our observed rates. As a final note, the lumped circuit approach is convenient, but not necessary. In the Appendix, we present a microscopic model that is valid for arbitrary ion-electrode distances and reproduces Eq. 5 for all the traps considered here (and for all realistic traps where $d \gg \delta$, where $\delta$ is the skin depth of the electrode material at the trap secular frequency).

\section{Fluctuating patch-potential noise}

To derive the heating rate for the case of microscopic patch-potentials we use the following approximate model. We assume that the trap electrodes form a spherical conducting shell of radius $a$ around the ion. Each of the patches is a disc on the inner surface of the sphere with radius $r_{p} \ll a$ and electric potential noise $V_{p}(\omega)$. Alternatively, each patch is assumed to have power noise spectral density $S_{V}(\omega)$. The electric field noise at the ion due to a single patch is $E_{p}(\omega)=-3 V_{p}(\omega) r_{p}^{2} / 4 a^{3}$ in the direction of the patch. There are $N \approx 4 C a^{2} / r_{p}^{2}$ such patches distributed over the sphere with coverage $C \leq 1$. Averaging over a random distribution of patches on the sphere, we find that the power spectral density of the electric field at the ion (along a single direction) is

$$
S_{E}(\omega)=N\left(\frac{\partial E_{p}(\omega)}{\partial V_{p}(\omega)}\right)^{2} S_{V}(\omega)=\frac{3 C S_{V}(\omega) r_{p}^{2}}{4 a^{4}} .
$$

This gives a heating rate

$$
\dot{\bar{n}}_{\mathrm{P}}=\frac{3 q^{2} C r_{p}^{2} S_{V}\left(\omega_{m}\right)}{16 m \hbar \omega_{m} d^{4}},
$$

in which the association $d \sim a$ is made. Note the difference in scaling with electrode size between Eqs. 5 and 17. The thermal electronic noise model gives a scaling $\dot{\bar{n}}_{\mathrm{R}} \propto d^{-2}$, while

the patch-potential model gives $\dot{\bar{n}}_{\mathrm{P}} \propto d^{-4}$. In fact, a $d^{-4}$ dependence also arises from a random distribution of fluctuating charges or dipoles. 


\section{MEASUREMENTS}

\section{A. Measuring the heating rate}

To determine the heating rate, we first cool the ion to near the ground state. In sufficiently strong traps, this is achieved simply by laser cooling with light red-detuned from a fast cycling transition $\left(\gamma \approx \omega_{m}\right.$, where $\gamma$ is the radiative linewidth of the upper state) propagating in a direction such that its $k$-vector has a component along the direction of the mode of interest. In weaker traps, additional sideband Raman cooling is utilized to cool to the ground state [3]. Typical starting values of $\bar{n}$, the average number of thermal phonons in the mode of interest, are between 0 and 2.

After cooling and optically pumping the ion to its internal ground state (denoted $|\downarrow\rangle$ ), we drive Raman transitions between atomic and motional levels [2 《4. Tuning the Raman difference frequency $\Delta \omega$ to the $k^{t h}$ motional blue sideband (bsb) at $\Delta \omega=\omega_{0}+k \omega_{m}$ drives the transition $|\downarrow\rangle|n\rangle \leftrightarrow|\uparrow\rangle|n+k\rangle$ where $|\uparrow\rangle,|\downarrow\rangle$ refer to the internal (spin) states of the atom that are separated by $\omega_{0}$. The $k^{t h}$ red sideband (rsb) at $\Delta \omega=\omega_{0}-k \omega_{m}$ drives $|\downarrow\rangle|n\rangle \leftrightarrow|\uparrow\rangle|n-k\rangle$. The measurement utilizes asymmetry in the strengths of the red and blue motional sidebands to extract $\bar{n}$. The strengths of the sidebands are defined as the probability of making a transition $|\downarrow\rangle \leftrightarrow|\uparrow\rangle$, which depends on the occupation number of the motional levels. The strengths are probed by a Raman pulse of duration $t$ tuned to either $k^{\text {th }}$ sideband. The probability $P_{\downarrow}$ of remaining in $|\downarrow\rangle$ after probing is measured and the strengths $I_{k}^{\mathrm{rsb}}=1-P_{\downarrow, \mathrm{rsb}}$ and $I_{k}^{\mathrm{bsb}}=1-P_{\downarrow, \mathrm{bsb}}$ are extracted. For thermal motional states, the strengths of the red and blue sidebands are related by [3,8]

$$
\begin{aligned}
I_{k}^{\mathrm{rsb}} & =\sum_{m=k}^{\infty} P_{m} \sin ^{2} \Omega_{m, m-k} t \\
& =\left(\frac{\bar{n}}{1+\bar{n}}\right)^{k} \sum_{m=0}^{\infty} P_{m} \sin ^{2} \Omega_{m+k, m} t \\
& =\left(\frac{\bar{n}}{1+\bar{n}}\right)^{k} I_{k}^{\mathrm{bsb}}
\end{aligned}
$$

where $\Omega_{m+k, m}=\Omega_{m, m+k}$ is the Rabi frequency of the $k^{t h}$ sideband between levels $m$ and 
$m+k$, and $P_{m}=\bar{n}^{m} /(1+\bar{n})^{m+1}$ is the probability of the $m^{\text {th }}$ level being occupied for a thermal distribution of mean number $\bar{n}$. The ratio of the sidebands $R_{k} \equiv I_{k}^{\mathrm{rbb}} / I_{k}^{\mathrm{bsb}}$ is independent of drive time $t$ and immediately gives the mean occupation number $\bar{n}$,

$$
\bar{n}=\frac{\left(R_{k}\right)^{1 / k}}{1-\left(R_{k}\right)^{1 / k}},
$$

which is valid even if the Lamb-Dicke criterion is not satisfied. In principle, $k$ should be chosen to be the positive integer nearest to $\bar{n}$ in order to maximize sensitivity. In practice we use $k=1,2$, or 3 in most cases. Note that Eq. 11 is valid only for thermal states; this is adequate since Doppler cooling leaves the motion in a thermal state [4.20], as does any cooling to near the ground state.

In order to determine the heating rate $\dot{\bar{n}}$, delays with no laser interaction are added between the cooling cycle and the probing cycle. An example of a data set at a fixed trap secular frequency is shown in Fig. 1. The error bars are determined as follows: The raw data of Raman scans over the sidebands (such as is those shown in the insets of Fig. 1) are fit to Gaussians, from which the depths of the sidebands are extracted, with error on the parameter estimate calculated assuming normal distribution of the data. The errors from the rsb and bsb strengths are propagated through Equation $\square$ for an error on $\bar{n}$. The error bars shown are one-sigma, and include only statistical factors. These errors are incorporated in the linear regression to extract $\dot{\bar{n}}$ with appropriate error. Many such data sets are taken for various types of traps and at different secular frequencies.

\section{B. The traps}

The measurements of heating rate in this paper extend over a five year period and utilize six different traps. The traps are summarized in Table $\mathbb{6}$. The traps are described in the references listed; here only a brief discussion is included. The "ring" traps are approximate quadrupole configurations consisting of a flat electrode (125 $\mu \mathrm{m}$ thick) with a hole drilled

through it (the ring) and an independent "fork" electrode (100 $\mu$ m thick) that forms endcaps 
on either side of the electrode, centered with the hole, similar to the trap shown in Fig. 2. In trap 1, the ring and endcap electrodes are at the same average potential; in traps 2 and 3 a static bias field could be added between the fork and ring to change the distribution of binding strengths along the three principle axes of the trap. The size of these traps is stated as the hole radius, with the endcaps spacing approximately $70 \%$ of the hole diameter. For the elliptical ring trap (trap 2) the stated size is the radius along the minor axis and the aspect ratio is $3: 2$; the fork tines are parallel to the major axis of the ellipse. Traps $3 \mathrm{a}$ and $3 \mathrm{~b}$ were drilled into a single flat electrode with a single graded fork electrode (see Fig. 2). The rings are circular and the size stated is the radius. This was the trap used for the size-scaling measurements. The heating in all of the ring traps was measured in a direction in the plane of the ring electrode, parallel to the tines of the fork electrode. Traps 4,5 and 6 are similar linear traps with geometry indicated in Fig. 3. Trap 6 was made slightly larger than traps 4 and 5 by increasing the space between the two electrode wafers. Heating was measured along the axial direction, which has only a static confining potential. The size quoted in Table [ for the linear traps is the distance between the ion and the nearest electrode. All traps are mounted at the end of a coaxial $\lambda / 4$ resonator for rf voltage buildup [21]. Typical resonator quality factors are around 500 and rf voltage at the open end is approximately $500 \mathrm{~V}$ with a few watts of input power. In all traps except for trap $3 \mathrm{a}$ and $3 \mathrm{~b}$ the resonator is inside the vacuum chamber with the trap. In traps $3 \mathrm{a}$ and $3 \mathrm{~b}$, the resonator is outside the chamber, with the high-voltage rf applied to the trap through a standard vacuum feedthrough.

Since we believe that surface effects are an important factor in heating, we cleaned the electrode surfaces before using a trap. When trap electrodes were recycled, they were first cleaned with $\mathrm{HCl}$ in order to remove the Be coating deposited by the atomic source. For the molybdenum traps an electro-polish in phosphoric acid was then used. For the beryllium electrodes electro-polishing in a variety of acids was ineffective, so abrasive polishing was used. Finally, the traps were rinsed in distilled water followed by methanol. The gold electrodes of the linear traps were cleaned with solvents after being evaporatively deposited on their alumina substrates. The time of exposure of clean trap electrodes to the atmosphere 
before the vacuum chamber was evacuated was typically less than one day. The traps were then vacuum baked at $\sim 350^{\circ} \mathrm{C}$ for approximately three days.

\section{Data}

Our longest-term heating measurements were made on trap 1. In Figure t we plot the heating rate as a function of date of data acquisition for a fixed trap frequency (11 MHz). The heating rate is on the order of 1 quantum per millisecond with a basic trend upwards of $\sim 1$ quantum per millisecond per year. Over this time the electrodes were coated with Be from the source ovens, but beyond this, nothing was changed in the vacuum envelope, which was closed for this entire period of time. The cause of the increase in heating rate is unknown, but may be related to increased Be deposition on the electrodes. Be plating on the trap electrodes could be a source of patch-potential noise.

Figure 5 shows heating rates in the linear traps (trap 4,5 and 6) and the elliptical ring trap (trap 2) as a function of trap secular frequency. The frequency dependence of the

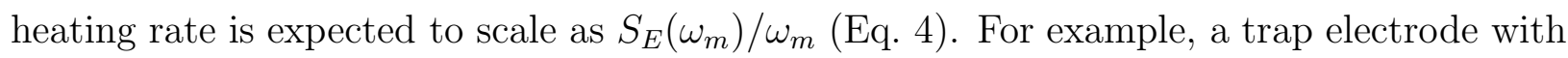

a flat noise spectrum $\left(S_{E}(\omega)=\right.$ constant $)$ will have a heating rate that scales as $\omega_{m}^{-1}$. The actual spectrum of fluctuations is impossible to know a priori, but in principle the data can be used to extract a spectrum over a limited frequency range given the model leading to Eq. 田. For the three linear traps, the heating rate data are most consistent with a $\omega_{m}^{-2}$ scaling, implying $S_{E} \propto \omega^{-1}$. This does not greatly assist in identifying a physical mechanism for the heating. For example, pure Johnson noise will have a flat spectrum, low-pass-filtered Johnson noise will have a spectrum that decreases with increasing frequency, and the spectrum of fluctuations in the patch-potential case is entirely unknown. In addition to the theoretical ambiguity, there is evidence in other data sets of different frequency scalings (though they are always power-law scalings). This measurement certainly cannot be used to pinpoint a heating mechanism; it is presented here only for completeness.

The data of Figure 5b provide a first indication of the scaling of the heating rate with 
trap size. Trap 6 is about 1.3 times larger than trap 5, while its heating rate (at $10 \mathrm{MHz}$ ) was a factor of 3 slower. This indicates that the dependence of heating rate on trap size is stronger than $d^{-2}$, but is consistent with $d^{-4}$. Of course, this comparison is to be taken with some caution, since these are two separate traps measured several weeks apart, and therefore likely had different microscopic electrode environments. However, a comparison is warranted since the traps were identical apart from their sizes. In particular, all the associated electronics was the same and the rf drive voltage was very nearly the same. In fact, the rf voltage was slightly larger for the measurements on trap 6, which showed the lower heating rate. This is important to note because we observe a slight dependence of the heating rate on the applied rf trapping voltage. Though we have only a qualitative sense of this dependence at present, it seems that heating rates increase with rf voltage, up to a point, at which the effect levels off. This rf-voltage dependence is observed along directions where the ion is confined both by static fields and by pondermotive fields. It may not be unreasonable that the increased rf voltage increases the intensity of the noise source (possibly due to an increase of temperature of the electrodes), even when it does not affect the trap secular frequency, as in the axial direction of the linear traps.

Trap 3 was designed to give a controlled measure of the heating rate as a function of trap size, while all other parameters were held fixed. The trap electrodes were made from the same substrates, the electrodes were subjected to the same pre-use cleaning, the traps were in the same vacuum envelope, driven by the same rf electronics (simultaneously) and data for both traps were acquired with minimal delay. For direct comparison at the same secular frequency in both traps, it was necessary to change the applied rf voltage since $\omega_{m} \propto 1 / d^{2}$. (A static bias between ring and endcap can be added, as discussed above, but this was not sufficient to measure heating at identical secular frequencies for the same rf drive.) There are two data sets to be discussed for this trap, shown in Figure 6 .

In the first set, shown in Figure 6a, we have data points at two different secular frequencies for the "small trap" (trap 3a) and one point for the "big trap" (trap 3b). The heating rates of the small trap are comparable to the heating rates for other traps and show a $\omega_{m}^{-1}$ 
scaling of the heating rate. The single point on the big trap is at a lower secular frequency, yet has a much slower heating rate. In fact, if we extrapolate the data from the small trap to the same secular frequency (using $\omega_{m}^{-1}$ ), the heating rate is over an order of magnitude lower in the big trap. The ratio of the heating rate in the small trap to that of the big trap is $20 \pm 6$. This is a much stronger scaling than that predicted by a Johnson noise heating mechanism (Eq. 5 predicts a $d^{2} \sim 4.8$ scaling), but is consistent with the scaling in the patch-potential case (Eq. 7 predicts a $d^{4} \sim 23$ scaling). When these data are used to predict an exponent for the size-scaling, the result is $d^{3.8 \pm 0.6}$.

For the second data set, shown in Figure $6 \mathrm{~b}$, the trap was removed from the vacuum enclosure, given the usual cleaning (as discussed above), and replaced for the measurements. In this data set, the trap behaved quite differently from all other traps, with heating rates significantly below those of other traps. Also, $S_{E}$ must have been a strong function of $\omega$ for this trap since the scaling with trap frequency was rather pronounced. The scaling with size was also strong: the heating rate was 16,000 times smaller in the big trap. When these data are used to predict an exponent for the size-scaling, the result is $d^{12 \pm 2}$. Needless to say, it is difficult to draw general conclusions from the data for this particular trap, but the difference in heating rates between the two traps seems to strongly indicate, again, that Johnson noise is not the source of the heating. We cannot be sure why this trap had such anomalous heating behaviour, but we speculate that it is due to a less-than-usual deposition of Be on the trap electrodes prior to the measurements, because the trap loaded with minimal exposure to the Be source atomic beam.

At this point it is useful to compare the present results to heating rates in other experiments. There are two other measurements. The first was done with ${ }^{198} \mathrm{Hg}^{+}[10]$. For that experiment $\omega_{m} / 2 \pi \approx 3 \mathrm{MHz}$ and $d \approx 450 \mu \mathrm{m}$ and the heating rate was $0.006 / \mathrm{ms}$. Accounting for scalings with trap frequency $\left(\omega_{m}^{-1}\right)$ [23] and mass $\left(m^{-1}\right)$, these results are consistent with the present results for a size-scaling of $d^{-4}$. Another measurement has been made with ${ }^{40} \mathrm{Ca}^{+}$[5]. For that experiment $\omega_{m} / 2 \pi \approx 4 \mathrm{MHz}$ and $d \approx 700 \mu \mathrm{m}$ and the heating rate was $0.005 / \mathrm{ms}$. Compared to the present experiments and the $\mathrm{Hg}$ experiment, this is 
also consistent with a $d^{-4}$ scaling, although it is certainly unlikely that all systems had the same patch field environment.

\section{CONCLUSIONS AND OUTLOOK}

We have measured heating from the ground state of trapped ions in different traps. The magnitude of heating rates and the results of the size-scaling measurements are inconsistent with thermal electronic noise as the source of the heating. The results do not indicate any strong dependence on trap-electrode material or on the type of trap potential (pondermotive or static). The rf voltage applied to the electrodes may play a role in heating, in as much as it may have an influence on patch-potentials.

Since we have not identified the mechanism for the observed heating, it is difficult to say what path should be taken to correct it. If fluctuating patch-potentials on the surface of the electrodes are the cause, then further cleaning may be appropriate. Additionally, better masking of the the trap electrodes from the Be source ovens may help.

The results coupled with those of other experiments [10,5], strongly indicate that bigger traps have smaller heating rates. This is not a surprise, but the strength of the scaling may be. With little sacrifice in the trap secular frequency (which ultimately determines the fastest rate of coherent manipulation) a dramatic decrease in the heating rate vs. logic gate speed appears possible using larger traps.

We acknowledge support from the U. S. National Security Agency, Office of Naval Research and Army Research Office. We thank Chris Langer, Pin Chen, and Mike Lombardi for critical readings of the manuscript.

\section{APPENDIX: THERMAL ELECTRIC FIELDS}

We are interested in the thermal electric field power spectral density $S_{E_{i}}(\boldsymbol{r}, \omega)$ generated from a specified volume of conductor. The conductor can be decomposed into a web of resistors each carrying current spectral density $S_{I_{i}}=4 k_{B} T / R_{i}$ (where we assume $k_{B} T \gg$ 
$\hbar \omega)$. The resistance along the $i t h$ direction of an infinitesimal volume element is $R_{i}=$ $d l /(\sigma d A)$, where $\sigma$ is the conductivity, $d l$ is the length along $i$ and $d A$ is the cross-sectional area. A Fourier component of current $I_{i}(\omega)$ through the volume $d V=d l d A$ gives rise to an electric dipole $P_{i}(\omega)=I_{i}(\omega) d l / \omega$, thus the equivalent spectral density of electric dipole of the infinitesimal resistor is isotropic: $S_{P_{i}}(\omega)=4 k_{B} T \sigma d V / \omega^{2}$.

The electric field from an electric dipole $\boldsymbol{P}\left(\boldsymbol{r}^{\prime}, \omega\right)$ oscillating at frequency $\omega$ and position $\boldsymbol{r}^{\prime}$ is

$$
E_{i}(\boldsymbol{r}, \omega)=\sum_{j=x, y, z} P_{j}\left(\boldsymbol{r}^{\prime}, \omega\right) G_{i j}\left(\boldsymbol{r}, \boldsymbol{r}^{\prime}, \omega\right) .
$$

In this expression, $G_{i j}\left(\boldsymbol{r}, \boldsymbol{r}^{\prime}, \omega\right)$ is a Green function matrix, representing the $i t h$ component of electric field at position $\boldsymbol{r}$ due to the $j$ th component of a point dipole at $\boldsymbol{r}^{\prime}$ which satisfies the appropriate boundary conditions of the geometry. The electric field spectral density at position $\boldsymbol{r}$ is an integral over the dipoles in the conductor volume:

$$
S_{E_{i}}(\boldsymbol{r}, \omega)=\frac{4 k_{B} T}{\omega^{2}} \int \sigma\left(\boldsymbol{r}^{\prime}\right) \sum_{j=x, y, z}\left|G_{i j}\left(\boldsymbol{r}, \boldsymbol{r}^{\prime}, \omega\right)\right|^{2} d V^{\prime} .
$$

The Green function satisfies $G_{i j}\left(\boldsymbol{r}, \boldsymbol{r}^{\prime}, \omega\right)=G_{i j}\left(\boldsymbol{r}^{\prime}, \boldsymbol{r}, \omega\right)$, so the above integral can be interpreted as the Ohmic power absorbed by the conductor from the electric fields generated by a point dipole at position $\boldsymbol{r}$. By energy conservation, this must be equivalent to the timeaveraged power dissipated by a point dipole at $\boldsymbol{r}$, which is related to the imaginary part of the Green function matrix $G_{i j}(\boldsymbol{r}, \boldsymbol{r}, \omega)$ [24]. This simplifies Eq. (13), leaving the fluctuationdissipation theorem

$$
S_{E_{i}}(\boldsymbol{r}, \omega)=\frac{2 k_{B} T}{\omega} \sum_{j=x, y, z} \Im m G_{i j}(\boldsymbol{r}, \boldsymbol{r}, \omega) .
$$

Agarwal solved Maxwell's equations for $G_{i j}\left(\boldsymbol{r}^{\prime}, \boldsymbol{r}, \omega\right)$ for the simple geometry of an infinite sheet of conductor filling the space $z \leq 0$ with the conductor-vacuum interface in the $z=0$ plane [25]. Although this idealized geometry is far from any real ion trap electrode structure, rough scalings of the thermal fields can be relevant to real ion trap geometries. From Ref. [25], the Green function matrix for this problem is diagonal with axial $(z)$ and radial $(\rho)$ components 


$$
\begin{gathered}
G_{z z}(z, z, \omega)=G^{f r e e}(\omega)+i \int_{0}^{\infty} \frac{q^{3}}{w_{0}}\left(\frac{w_{0} \varepsilon-w}{w_{0} \varepsilon+w}\right) e^{2 i w_{0} z} d q \\
G_{\rho \rho}(z, z, \omega)=G^{f r e e}(\omega)-\frac{i}{2} \int_{0}^{\infty} q\left[w_{0}\left(\frac{w_{0} \varepsilon-w}{w_{0} \varepsilon+w}\right)+\frac{k^{2}}{w_{0}}\left(\frac{w-w_{0}}{w+w_{0}}\right)\right] e^{2 i w_{0} z} d q,
\end{gathered}
$$

In the above expressions, $\varepsilon(\omega)=\varepsilon_{0}+i \sigma / \varepsilon_{0} \omega$ is the dielectric function of the conductor (in the low frequency limit), $k=\omega / c$, and wavevectors $w_{0}$ and $w$ (generally complex) are defined by $w_{0}^{2}=k^{2}-q^{2}$ and $w^{2}=k^{2} \varepsilon-q^{2}$ with $\Im m w_{0} \geq 0$ and $\Im m w \geq 0$. The free space Green's function $G^{\text {free }}(\omega)$ has imaginary part $\Im m G^{\text {free }}(\omega)=k^{3} / 6 \pi \varepsilon_{0}$ and gives rise to the isotropic free space blackbody electric field fluctuations when substituted into Eq. (14).

The above integrals are significantly simplified in the "quasi-static" limit, where $k z \ll 1$ and the conductivity is sufficiently high so that $k \delta \ll 1$, where $\delta=\sqrt{2 c^{2} \varepsilon_{0} / \omega \sigma}$ is the skindepth of the conductor. Despite these conditions, no restriction is placed on the value of $z / \delta$. We break the above integrals into two pieces. The first piece $\int_{0}^{k}$ has $q \leq k$ with $w_{0}$ real. In the quasi-static limit, this piece can be shown to cancel the free space contribution to the transverse Green function $\Im m G_{\rho \rho}(z, z, \omega)$ while doubling the free space contribution to the axial Green function $\Im m G_{z z}(z, z, \omega)$. Physically, the presence of the conductor negates the transverse free space blackbody field while it doubles the axial blackbody field due to a near-perfect reflection. The second piece of the integrals $\int_{k}^{\infty}$ has $q \geq k$ with $w_{0}$ imaginary. These pieces of the integral can be solved to lowest order in $k z$ and $k \delta$. Combining terms and substituting the results into Eq. (14), the thermal electric field spectral density is

$$
\begin{gathered}
S_{E_{z}}(z, \omega)=\frac{2 k_{B} T \omega^{2}}{3 \pi \varepsilon_{0} c^{3}}+\frac{k_{B} T}{4 \pi \sigma z^{3}} \sqrt{\frac{1}{2}+\sqrt{\frac{1}{4}+\frac{z^{4}}{\delta^{4}}}} \\
S_{E_{\rho}}(z, \omega)=\frac{k_{B} T}{8 \pi \sigma z^{3}} \sqrt{\frac{1}{2}+\sqrt{\frac{1}{4}+\frac{z^{4}}{\delta^{4}}}} .
\end{gathered}
$$

These expressions show that the thermal electric field noise scales as $1 / z^{3}$ for $z \ll \delta$ [14], but scales as $1 / z^{2}$ for $z \gg \delta$ [8, [1]]. At large distances $z>\sqrt{\delta / k}$ (with $k z \ll 1$ ), the axial field noise settles toward twice the free space blackbody value while the radial field vanishes. 
This result is also reported in Ref. [26]. The behavior is shown in Fig. 7, where Eqs. (17) and (18) have been substituted into Eq. \&, giving the expected thermal heating rate for a ${ }^{9}$ Be ion trapped with molybdenum electrodes at room temperature. Note that the predicted heating rate at trap sizes typical in our experiments is significantly slower than the 0.1-1 quanta/s rate predicted in $[8,[1]$. This difference comes from the choice of the value of the resistance in Eq. 5, which was chosen in 8, 11] as an absolute upper limit.

When interpreting these results, only the rough scaling should be considered. Realistic ion trap electrode geometries are more complicated than a single infinite conducting plane, involving a more closed electrode structure. This generally requires a full numerical solution to the relevant boundary value problem. Moreover, we are usually interested in the electric field fluctuations at the center of the trap, where these fluctuations will be substantially different from those above an infinite plate.

* Work of the US government. Not subject to US copyright.

$\dagger \quad$ quentint@boulder.nist.gov.

a present address: NIST, Gaithersburg, MD.

$b$ present address: Univ. Innsbruck, Austria.

c present address: Research Electro-optics, Boulder, CO.

$d$ present address: ILX Lightwave, Boulder, CO.

[1] J. I. Cirac and P. Zoller, Phys. Rev. Lett. 74, 4091 (1995).

[2] C. Monroe, D. M. Meekhof, B. E. King, W. M. Itano, D. J. Wineland, Phys. Rev. Lett. 75, $4714(1995)$.

[3] C. Monroe, D. M. Meekhof, B. E. King, S. R. Jefferts, W. M. Itano, D. J. Wineland, P. Gould, Phys. Rev. Lett. 75, 4011 (1995). 
[4] D. M. Meekhof, C. Monroe, B. E. King, W. M. Itano, D. J. Wineland, Phys. Rev. Lett. 76, $1796(1996)$.

[5] Ch. Roos, Th. Zeiger, H. Rohde, H. C. Nägerl, J. Eschner, D. Leibfried, F. Schmidt-Kaler, R. Blatt, Phys. Rev. Lett. 83, 4713 (1999).

[6] B. E. King, C. S. Wood, C. J. Myatt, Q. A. Turchette, D. Leibfried, W. M. Itano, C. Monroe, D. J. Wineland, Phys. Rev. Lett. 81, 1525 (1998).

[7] Q. A. Turchette, C. S. Wood, B. E. King, C. J. Myatt, D. Leibfried, W. M. Itano, C. Monroe, D. J. Wineland, Phys. Rev. Lett. 81, 3631 (1998).

[8] D. J. Wineland, C. Monroe, W. M. Itano,D. Leibfried,B. E. King,D. M. Meekhof, J. Res. Natl. Inst. Technol., 103. 259 (1998).

[9] C. J. Myatt, B. E. King, Q. A. Turchette, C. A. Sackett, D. Kielpinski, W. M. Itano, C. Monroe, D. J. Wineland, Nature 403, 269 (2000).

[10] F. Diedrich, J. C. Bergquist, W. M. Itano, D. J. Wineland, Phys. Rev. Lett. 62, 403 (1989).

[11] D. J. Wineland, C. Monroe,W. M. Itano, B. E. King, D. Leibfried, D. M. Meekhof, C. Myatt, C. Wood, Fortschr. Phys. 46, 363 (1998).

[12] S. K. Lamoreaux, Phys. Rev. A56, 4970 (1997).

[13] D. F. V. James, Phys. Rev. Lett. 81, 317 (1998).

[14] C. Henkel and M. Wilkens, Europhys. Lett. 47, 414 (1999).

[15] T.A. Savard, K.M. O'Hara, and J.E. Thomas, Phys. Rev. A 56, R1095 (1997).

[16] J. B. Camp, T. W. Darling, R. E. Brown, J. Appl. Phys. 69, 7126 (1991).

[17] C. Kleint, Annalen der Physik 10, 309 (1963).

[18] G. W. Timm and A. Van der Ziel, Physica 32, 1333 (1966). 
[19] D.J. Wineland and H.G. Dehmelt, J. Appl. Phys. 46, 919 (1975).

[20] S. Stenholm, Rev. Mod. Phys. 58, 699 (1986).

[21] S.R. Jefferts, C. Monroe, E.W. Bell, and D.J. Wineland, Phys. Rev. A51, 3112 (1995).

[22] C.A. Sackett, D. Kielpinski, B.E. King, C. Langer, V. Meyer, C.J. Myatt, M. Rowe, Q. A. Turchette, W. M. Itano, D. J. Wineland, and C. Monroe, unpublished (2000).

[23] There is no way to know if $\omega_{m}^{-1}$ is the proper way to scale for trap frequency, but it is a reasonable first assumption.

[24] L.D. Landau and E.M. Lifshitz, Statistical Physics, 3rd edition Part I (Pergamon, Oxford, 1980), Chapter XII.

[25] G.S. Agarwal, Phys. Rev. A11, 230 (1975).

[26] C. Henkel, S. Potting, M. Wilkens, Appl. Phys. B 69, 379 (1999). 


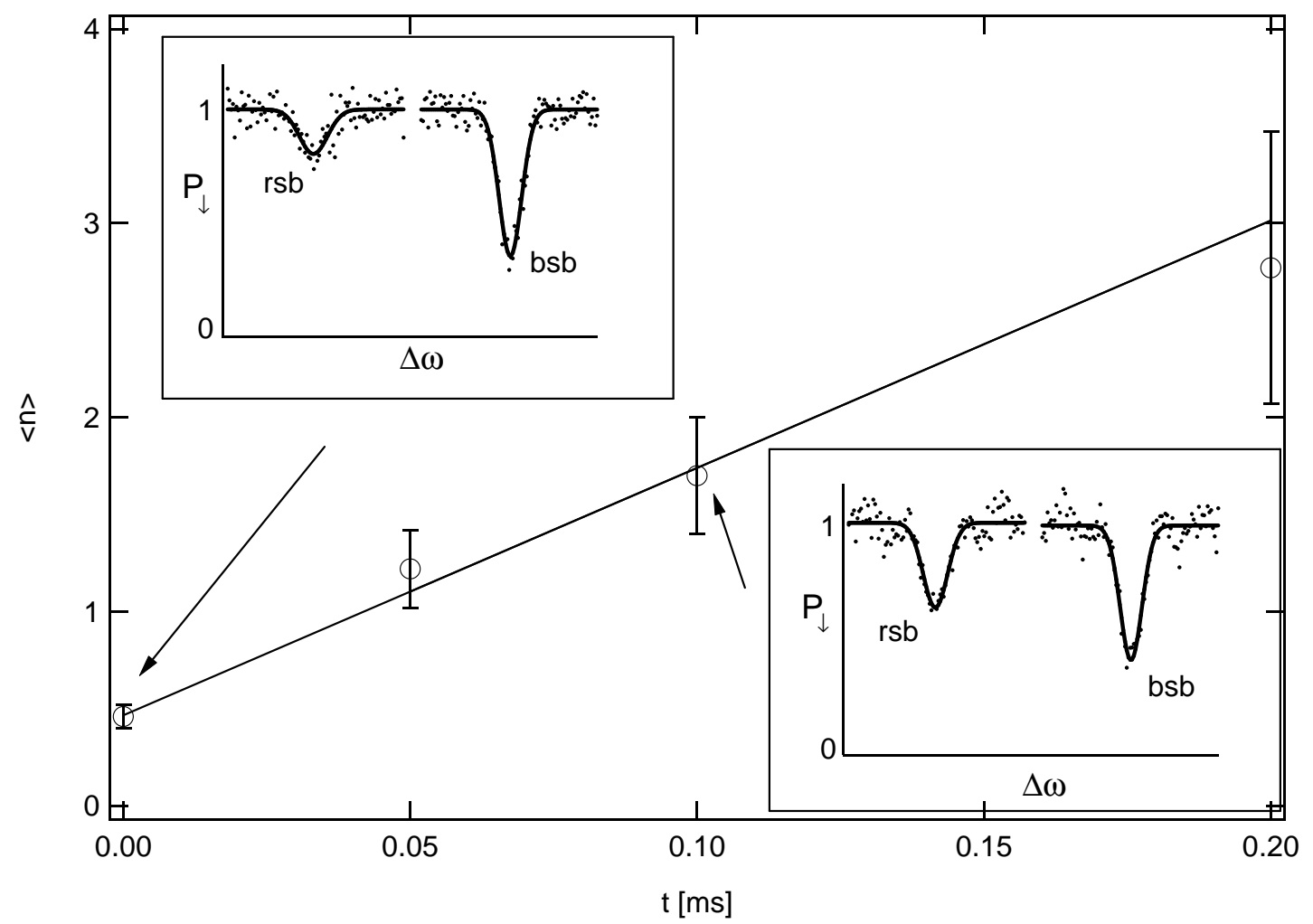

FIG. 1. An example of heating rate data. The main graph shows $\bar{n}(\equiv\langle n\rangle)$ vs $t$, the delay between cooling and probing. The insets show Raman spectra from which $\bar{n}$ is extracted, according to Eq. 11. For the insets, $P_{\downarrow}$ is the probability that the ion remains in the $|\downarrow\rangle$ state after application of a Raman probe of fixed duration with difference frequency $\Delta \omega$; rsb: red motional sideband, bsb: blue motional sideband. The sidebands shown are the 1st sidebands. The data are for trap 5 from Table 1 at $5 \mathrm{MHz}$ secular frequency and $\dot{\bar{n}}=12 \pm 2 / \mathrm{ms}$. 


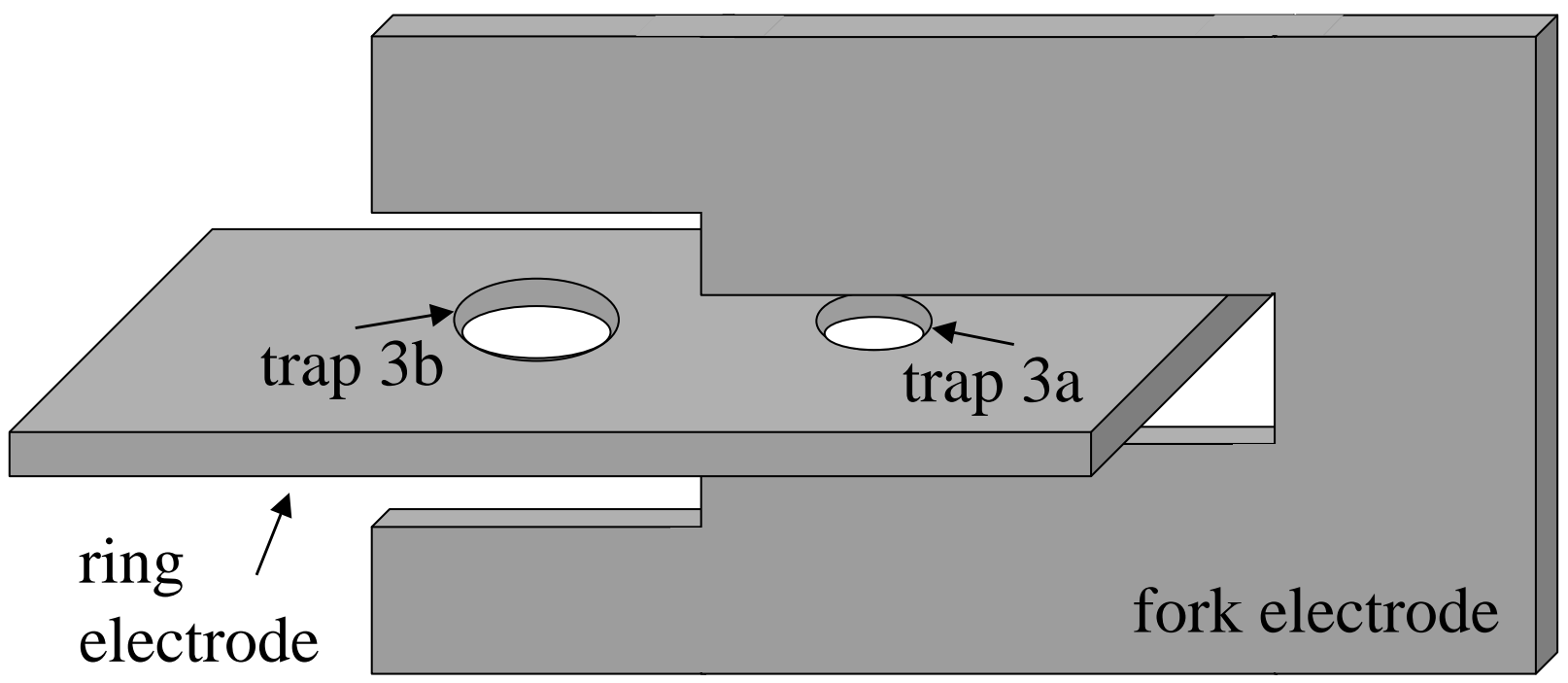

FIG. 2. Schematic diagram of the electrodes of trap 3 (from Table [1). The distance between traps $3 \mathrm{a}$ and $3 \mathrm{~b}$ is $1.7 \mathrm{~mm}$. Not to scale. 


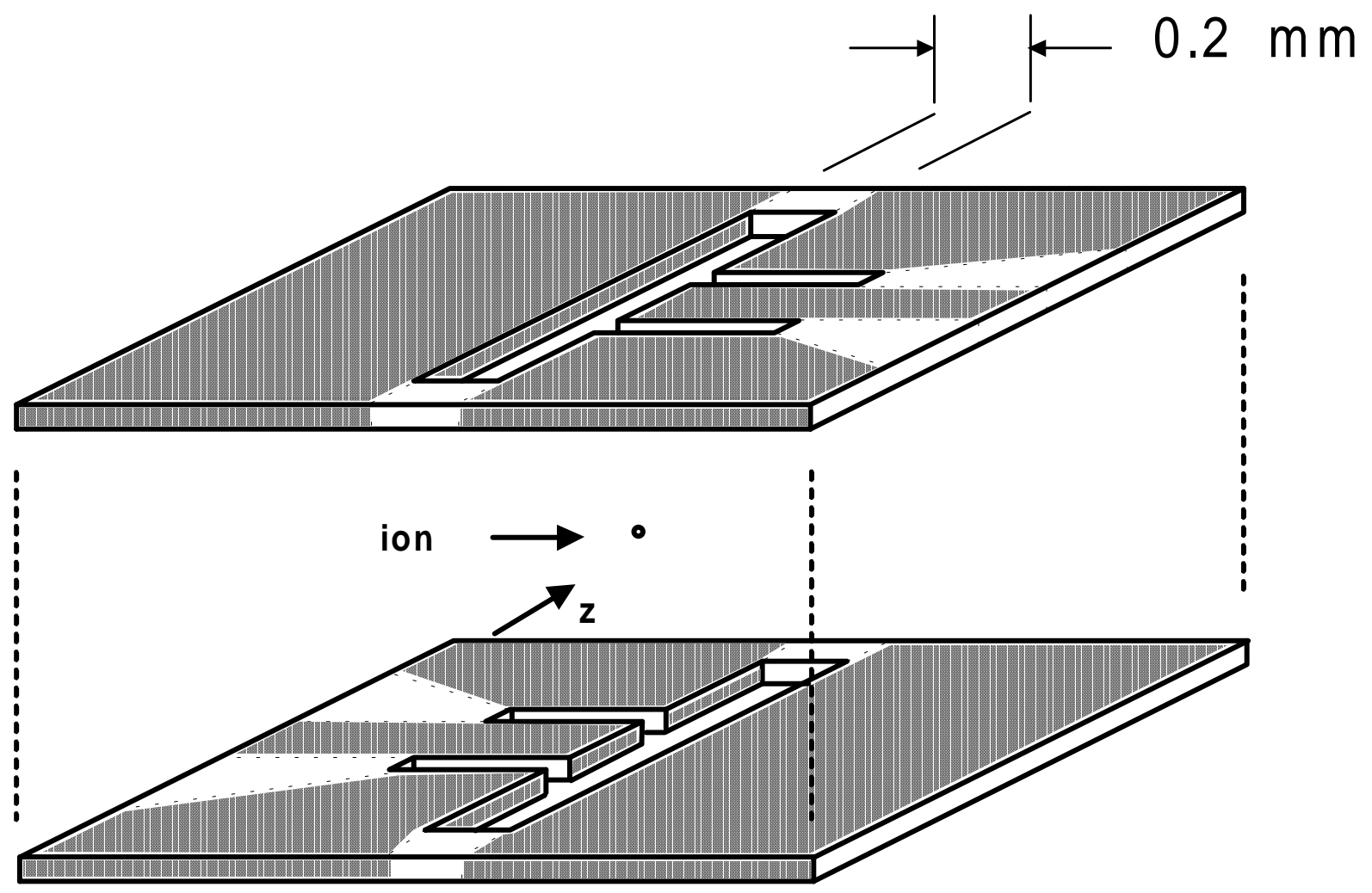

FIG. 3. Schematic diagram of the electrodes of the linear traps (traps 4, 5 and 6 from Table $\mathbb{1}$ ). The traps are formed by evaporating gold (approximately $0.75 \mu \mathrm{m}$ thick) on an alumina substrate. The outer segmented electrodes are the endcaps, while the long unbroken electrodes carry rf. The axial direction (labeled $z$ ) is parallel to the $\mathrm{rf}$ electrode. The two separate trap wafers are spaced by $200 \mu \mathrm{m}$ for traps 4 and 5 and $280 \mu \mathrm{m}$ for trap 6 (spacers not shown). Schematic diagram not to scale. 


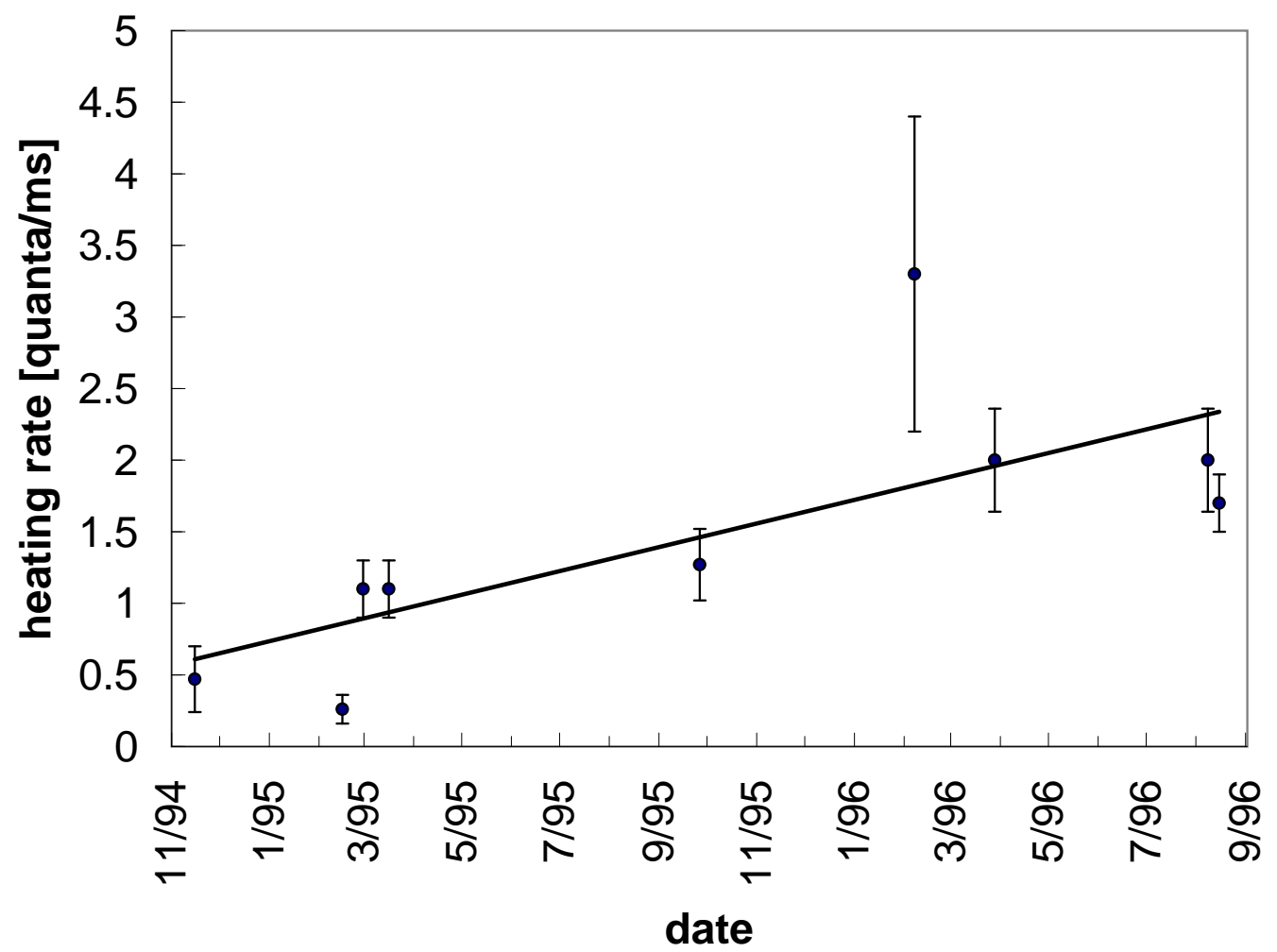

FIG. 4. Heating rate in ring trap 1 vs. time. The secular frequency for all measurements was $\sim 11 \mathrm{MHz}$. The solid line shows a trend which does not account for the weights of the data points. 

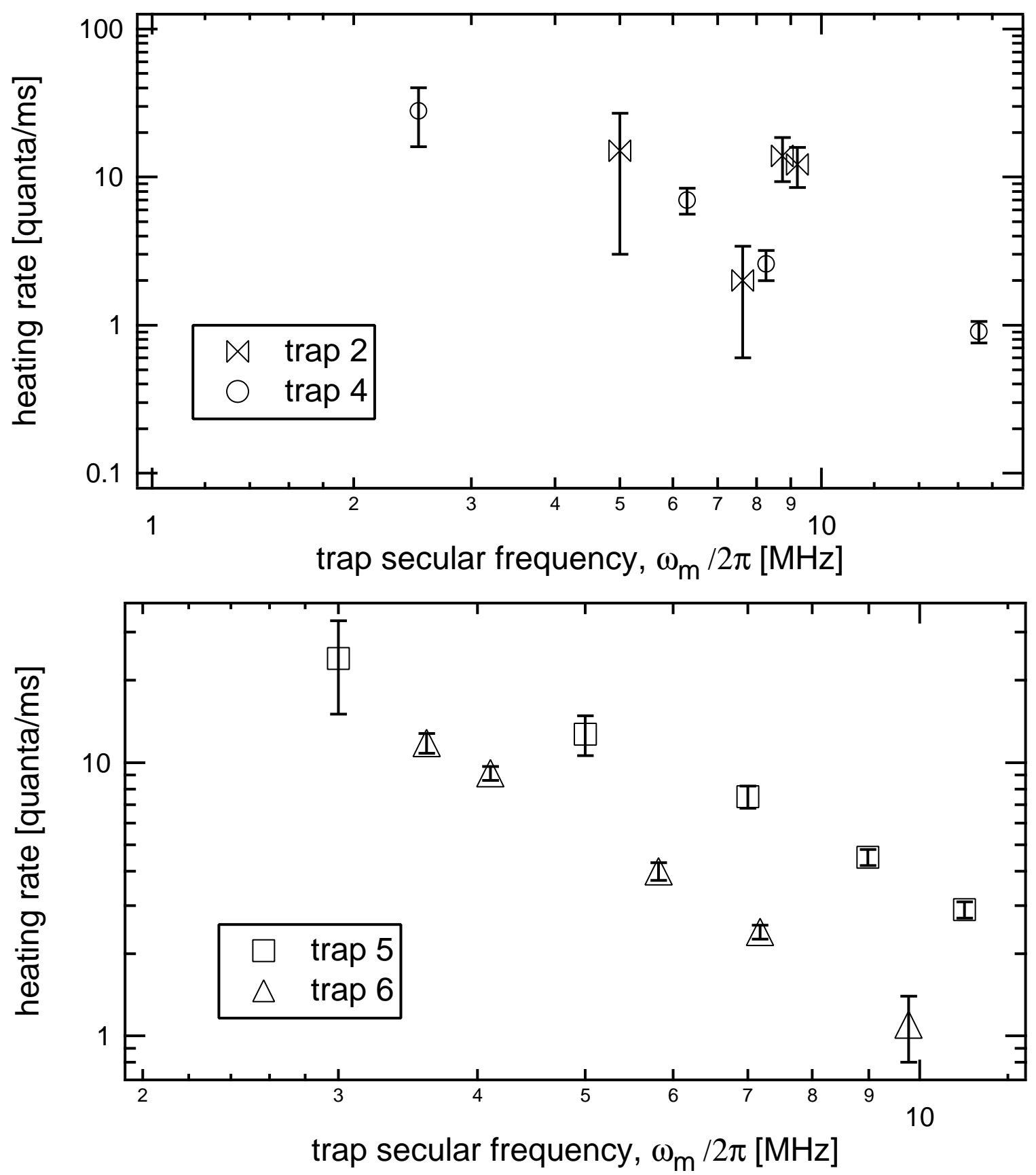

FIG. 5. Heating rates vs. trap secular frequency in a) the elliptical ring trap 2 and in the micro linear-trap 4 and in b) linear traps 5 and 6 . The only intended difference between traps 5 and 6 is the size. In all four data sets, the secular frequency was varied by changing a static potential only. 

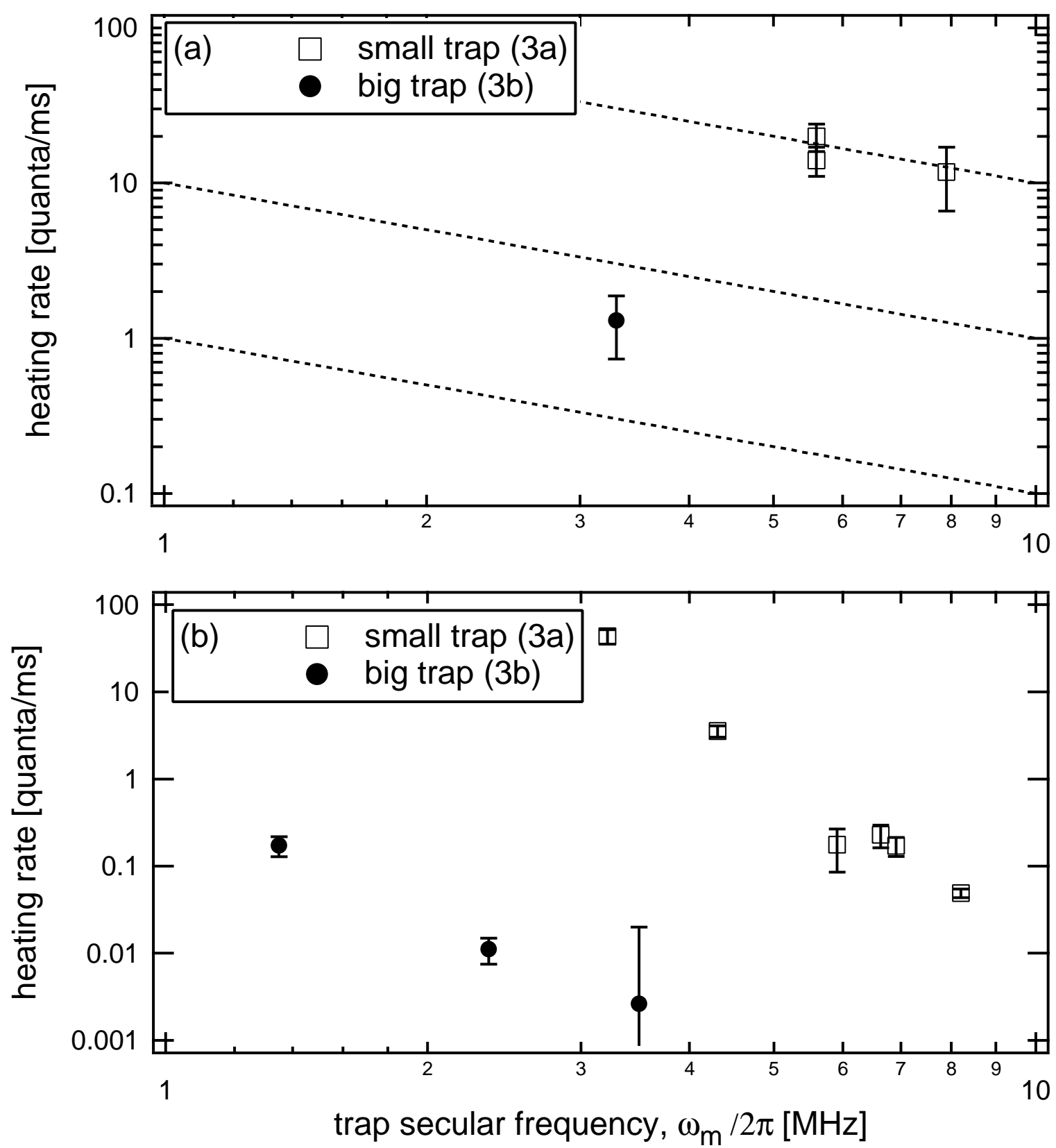

FIG. 6. Data from trap 3, showing heating rates vs. trap secular frequency. (a) Data set \#

1. The two points on the small trap at $\omega_{m} / 2 \pi=5.3 \mathrm{MHz}$ were taken with Raman cooling to $\bar{n}(t=0) \sim 0$ and with Doppler cooling only to $\bar{n}(t=0) \sim 2$. Note that they give comparable results, as they should. The dashed lines show a $\omega_{m}^{-1}$ scaling. (b) Data set \# 2. The small trap data were taken with an $\mathrm{rf}$ voltage of $\sim 400 \mathrm{~V}$ and the big trap with $\sim 600 \mathrm{~V}$. The secular frequency was changed by tuning the DC bias between fork and ring electrodes. 


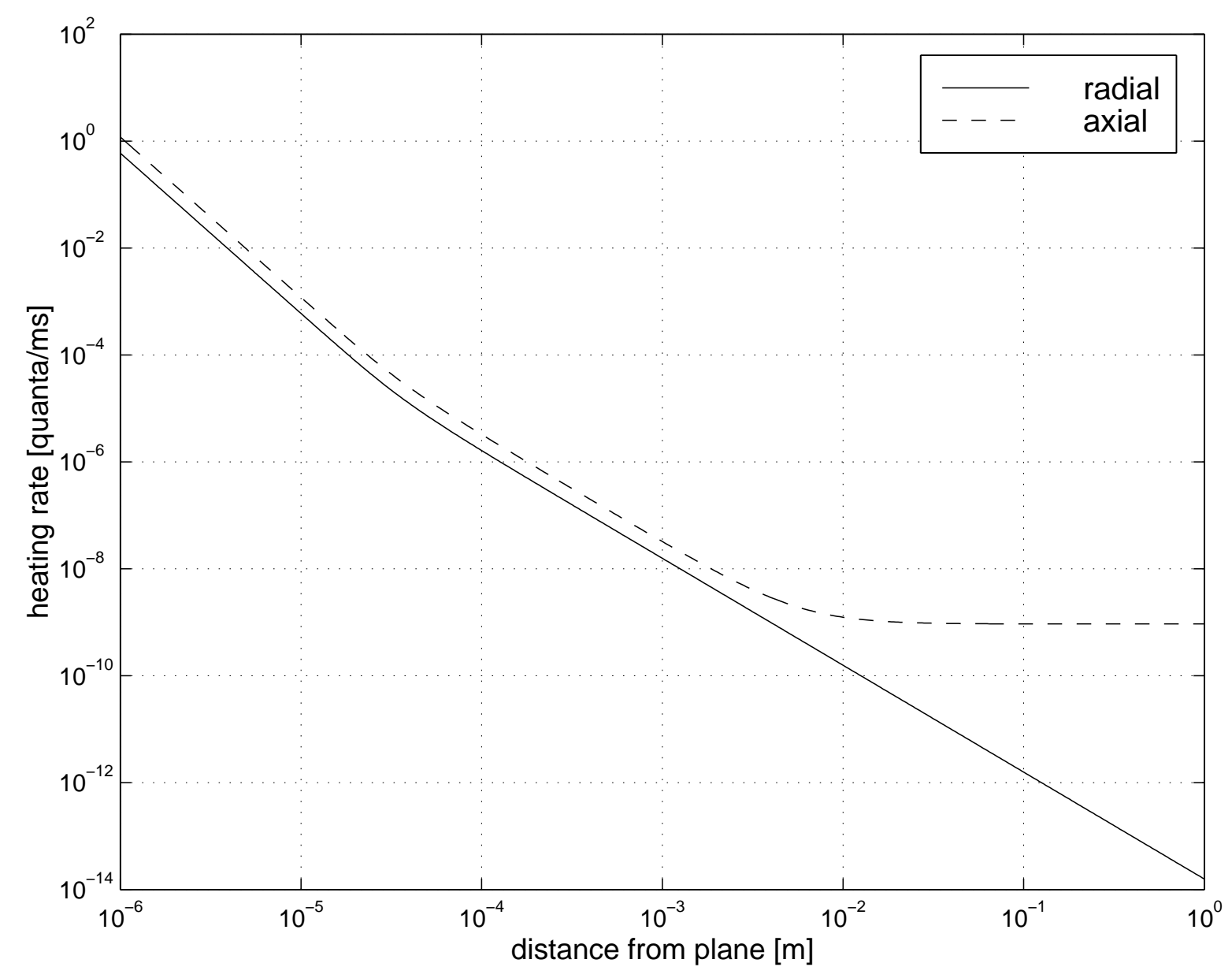

FIG. 7. Expected heating rate vs. distance from electrode for thermal electronic noise, from Eqs. (17), (18) and (41). The numerical parameters are those for ${ }^{9} \mathrm{Be}^{+}$with molybdenum electrodes at $10 \mathrm{MHz}$ secular frequency and room temperature. 


\begin{tabular}{|c|c|c|c|c|c|}
\hline trap & type & material & size $[\mu \mathrm{m}]$ & $\Omega_{T} / 2 \pi[\mathrm{MHz}]$ & $\dot{\bar{n}}\left[\mathrm{~ms}^{-1}\right]$ \\
\hline 1 & circular ring & Mo & 170 & 250 & 1 \\
\hline 2 & elliptical ring & $\mathrm{Be}$ & 175 & 250 & 10 \\
\hline $3 \mathrm{a}$ & circular ring & Mo & 175 & 150 & $10,10^{-2}$ \\
\hline $3 b$ & circular ring & Mo & 395 & 150 & $0.5,10^{-5}$ \\
\hline 4 & linear & $\mathrm{Au}$ & 280 & 150 & 2.3 \\
\hline 5 & linear & $\mathrm{Au}$ & 280 & 230 & 3.5 \\
\hline 6 & linear & $\mathrm{Au}$ & 365 & 230 & 1.1 \\
\hline
\end{tabular}

TABLE I. Summary of traps. The size column is approximately the distance between the ion and the nearest electrode surface. $\Omega_{T}$ is the trap rf-drive frequency. The heating rate $(\dot{\bar{n}})$ is for a trap secular frequency of $10 \mathrm{MHz}$, which in the case of traps $3 \mathrm{a}$ and $3 \mathrm{~b}$ had to be extrapolated from data at lower trap secular frequencies. The two numbers quoted for $3 \mathrm{a}$ and $3 \mathrm{~b}$ are for two different versions of the trap. See the text for a further discussion of the parameters. 